\title{
IoT based model of healthcare for physiotherapy
}

\author{
Gabriela Postolache \\ Instituto de Telecomunicações \\ Lisboa, Portugal \\ Pedro Silva Girão \\ Instituto Superior Tecnico \\ Universidade de Lisboa \\ Instituto de Telecomunicações \\ Lisboa, Portugal
}

\author{
Octavian Adrian Postolache \\ Escola Superior de Arquitectura e \\ Tecnologias, ISCTE-IUL \\ Instituto de Telecomunicações \\ Lisboa, Portugal
}

\author{
José Miguel Dias Pereira \\ ESTSetúbal/IPS \\ Instituto de Telecomunicações \\ Setúbal, Portugal \\ Vitor Viegas \\ CINAV - Escola Naval \\ Instituto de Telecomunicações \\ Lisboa, Portugal
}

\begin{abstract}
Small and reliable devices that are used not only in clinics or hospital but also in home, give information on movements, activities or other relevant data on person health and functioning. The data acquired by these devices would increase the accessibility to healthcare services and quality of care, in a safe environment. There are scarce data related to integration of Internet of Things (IoT) technologies into information system for physiotherapy or motor rehabilitation. In this work it is presented a framework for IoT based information system for physiotherapy. The presented model for physiotherapy includes: the capacity of IoT based information system to receive inputs from different modalities; support for modularity and common communication technologies for IoT; gateway capabilities and/or edge computing; data storage and analysis in Server, Cloud Server or Microservices. Research is needed for better understanding what is the optimal model and architecture for IoT platforms targeting people with different types of disabilities, as well as an optimal universal design that may increase the quality of care for people with disability.
\end{abstract}

Keywords- health model, Internet of Things, physiotherapy

\section{INTRODUCTION}

During human civilizations evolution, different models existed for health care, namely: i) the biomedical model, based on the mechanist way to see the diseases, which focuses on physical or biological processes (i.e., physical phenomena or changes in molecular or cellular structure of the human body) without considering the role of social and environmental factors, or individual perspectives (the reactive healthcare services focused on treatments and less in prevention are mainly centered on biomedical model [1]); ii) the model of social determination of health [2]; the biopsychosocial model [3]; and the holistic model [4]. Efforts are being made to integrate the relationships between different components of healthcare, including considerations of the demographic, social, environmental, and epidemiologic determinants. New approach to healthcare has increasing relevance mainly in the western countries, where the burden of care, higher costs for health and social care, and a greater impact of co-morbidities is associated with ageing population and increased level of disability [5].

Health information systems (such as Electronic Health Records (HER), Electronic Medical Records (EMR), electronic orders and reporting, electronic prescribing, evidence-based decision support, and preventive disease registry) have promising to contribute for health care services continuity, increased quality and efficiency of health care, including services related with care information, care management, care coordination, and fiscal responsibility [6].
The importance of addressing the preservation of functional autonomy of individuals with moderate-to-sever impairment was underscore in several care model, in which social, technological or environmental aspects were considered in the healthcare systems. As accessibility and efficiency of health care system are influenced by numerous factors (i.e., redundant evaluations of healthcare services users; failure on, or not using standardized tools; inappropriate use of costly resources; inefficient communication between services; fragmented response to needs), several health care systems that take into consideration the social aspects of frail, disabled people were proposed. For instance, the Canadian PRISMA Integrated Service Delivery System includes among others features: a single-entry point; a case, individualized, management process; assessment instrument based on the client's functional autonomy; tools for better communication, in and between healthcare services [7]. The Cerner Millenium Electronic Health Records system developed by the Department of Defense for the Department of Veterans Affairs, U.S.A for people with disability is an information system for which the technological challenges and solutions are often discussed in scientific literature. This information system has proposed improvement in management of veterans' care by considering their social and healthcare needs [8-9]. Examples of health care models that consider integration of environmental information in decisions for optimal healthcare are: the Service's Sustainable Development Unit that was established in 2008, which focuses on achieving environmental, social and financial sustainability within England's healthcare system [10]; and the Australian Medical Association proposal for environmental sustainability that should include stakeholders as Doctors for Environment Australia, the Australia Healthcare and Hospital Association, The Royal Australian College of Physicians, and academics [11].

A series of reports and documents published by the Council of Europe have been proposed: improvements in the quality of life of people with disabilities, which should be based on a sound assessment of their situation, potential and needs; creation of activities that enable a good state of physical and mental health; development of supportive structures around persons with disabilities in need of extensive support; provision of quality of healthcare services for people with disabilities; development of programs and resources to meet the needs of persons with disabilities [5]. Evidences have shown that five to nine-fold savings would be obtained if physical and rehabilitation medicine is provided and that rehabilitation is effective in all phases of health conditions [12-13]. Interventions, which may be provided through tele-rehabilitation by highly specialized specialists, may produce important savings (as specialized rehabilitation is highly cost-efficient, especially for 
neurological patients). This may produce substantial savings in ongoing care costs, especially in high-dependency patients [14].

There is scarce information on different technologies that may be integrated in order to provide improvement in quality of care for frail, disabled people, particularly those living in areas with less accessibility to healthcare services. Moreover, there is a lot of data on different small devices that were developed in the last years that people, during neuro-motor rehabilitation both in clinics or hospital and in ambulatory setting, have benefited greatly from their use. In this work we investigated what and how the technologies for motor rehabilitation would be integrated using the Internet.

\section{INTERNET OF THINGS FOR PHYSIOTHERAPY - WHAT DEVICES}

Different devices/motor rehabilitation equipment are being developed for people with disabilities: human powered devices, such as body powered upper limb prostheses; exoskeleton systems; motorized orthotics; pediatrics robotics; robot assisted therapies; targeted muscle reinnervation techniques; technologies for wheeled mobility and seating devices; simulated and augmented environments; alternative human-machine interfaces; technologies for low vision, blindness and multisensory loss; mobile technologies to support health self-management in adolescents with disabilities; technologies for successful aging with disability; cognitive technologies; optical tracking technologies for motion analysis; wearable technologies, and interactive exercises such as exergames [15]. Internet of Things (IoT) technologies, achieved by connecting physical and virtual objects to other objects and to the Internet [16], may contribute for improving quality of life for people with disabilities. Considering the rapid progress and changes in communication technologies, middleware platforms, data storage recipients, human-machine interfaces, and artificial intelligence for data mining and data presentation, the designers and developers of IoT-based information systems for physiotherapy have to overcome many challenges.

In our research we identified several requirements for IoT-based information systems for physiotherapy: capacity to receive inputs from different modalities; support for modularity and common communication technologies; gateway capabilities and/or edge computing; data storage and analysis in a server, cloud server or microservices.

\section{Multi-Modalities Sensing, Modularity, AND SAFE CONNECTIVITY}

\section{A. Capacity to receive inputs from different modalities}

An IoT based system for people with disabilities should mainly recognize inputs from different modalities (i.e., keyboard, mouse, speech, pen touch, manual gestures, body part movements), combining them according to temporal and contextual constraints. It should be able to easily support the extensibility of the existing devices, the upgrades and the integration of new modules.

Different sensors can be used in applications for monitoring different characteristics of movements. Some examples of commercially available sensors are: accelerometers (e.g. Locometrix); inertial measurements units (e.g., Axivity Ax3, Xsens 3D Motion Sensors, Shimmer); force sensors included in force platforms (e.g.,
GaitRite, Teckscan, AMTI Accusway, Kistler), in insoles (Digit Sole Warm Series, Stridalyzer, Lechal Smart Insole), in shoes (e.g., Under Armour Speed Form Gemini, Adidas MyCoach Speed Cell), in socks (e.g., Sensoria); electromyography sensors (e.g., Triton DTS, Shimmer); and infrared sensors (e.g., Microsoft Kinect, Leap Motion). Gloves capable of capturing hands movements (particularly fingers) are also in development or already commercialized (e.g., 5DT Data Gloves, Neofect's RAPAEL Smart Glove, Saebo's Glove, Flint Rehab Music Glove). Devices that are able to capture information about upper or lower limbs motion, or trunk movements, in combination with vital signals, are also in development or commercially available (e.g., FitBit, Nokia Withings Activité, Adidas miCoach Smart Run, Moov Now and Moov HR, Garmen Forerunner, Zephyr Bioharness, Monica Novii Wireless Patch System).

Each device should be identified in a unique way by an identifier system having a large enough number of identifiers to support all current and future devices, including high-level naming strategies like URIs or URLs. The technique to identify the devices must be compliant to device standards and extensive across different platforms (e.g., MAC address and NOMs) [17]. At the first attempt of the connection, the system architecture should be able to provide tools and services for checking the capacity of the user's device according to the characteristics of the device required for its application (i.e., plug-and-play should be supported).

\section{B. Support for modularity and common communication technologies}

More than one billion people in the world today experience some form of disability and half of them cannot afford health care's [18]. Body impairments, activity limitations and participation restrictions are the main features that characterize people with disabilities. An impairment is a problem in body function or structure. An activity limitation is a difficulty encountered by an individual in executing a task or action while a participation restriction is a problem experienced by an individual in involvement in life situations [19]. The disabled people living in geographically dispersed regions generally experience barriers on accessing health facilities, health information or a rehabilitation equipment [18]. Considering that different type of devices, used by people with disabilities in different locations of the ecosystem could include old or new communication protocols, the information system based on IoT technologies should support different communication protocols.

The communication of data in IoT framework might be supported by different types of wired or wireless technologies such as CAN bus, Zig Bee, Bluetooth, Wi-Fi, LoRa. Cellular connectivity like LTE is expected to increase in the future, especially with $5 \mathrm{G}$ and NarowBand-IoT. There are several hardware platforms available for sensor network deployment such as TelosB, Mica, IRIS, Waspmote, XBee ZB module, LoRa, BTLE, Tiny-mesh, Wirepasmesh, and Xelba xOne Hub. The presence of buildings, volumetric restrictions, and other topographic constraints should be considered on the power of communications locations. Constrains in terms of power supply should also be considered, and different technologies for power supply ranging from rechargeable batteries, renewable power generators (e.g. solar panels or wind turbines) or power harvesting techniques should be included in a IoT based system for physiotherapy. 
Tailoring, customizing or personalizing the multidimensional information to suit specific needs of patients and therapists requires software that would manage heterogeneous information regarding the users' needs and preferences, the user environments, and the devices used for motor rehabilitation. Modularity, the flexibility to introduce new applications or devices into the information system, for functioning assessment or for therapy, should be considered. Software-defined, 5G networks and network-functionvirtualization, as well as automated scenario generation, are areas of research and innovation that may improve the service quality by ensuring that the exergames may be provided in an efficient, personalized and controllable way. In the $5 \mathrm{G}$ context, everything will tend to be offered as a service, whether it is infrastructures, platforms or software, following the cloud software-platform-infrastructure model. In this context, the development of exergames for motor rehabilitation goes beyond the focus of underlying infrastructure toward the stakeholders of the ecosystem (i.e., patients, therapists, family physicians, cloud service providers, device providers) including Service Level Agreements (SLA) management that ensure that Virtual Network Services (VNS) and Virtual Network Functions (VNF) are provided in an efficient and controllable way. Quality of Service (QoS) expectations are driving end-users to negotiate specific QoS levels with their service providers, and therefore make QoS provision and management a very important and challenging field in distributed environment.

The IoT node should communicate with back-end information systems and/or directly with other IoT node, in order to provide real-time data and run processes for data collection, data transmission, data validation and response to available information. Machine-to-Machine (M2M) should be used for this purpose using events and/or polling. IoT middleware platforms should support the implementation of software wrappers to facilitate the interoperability [17].

In order to exchange not only data, but information and features related to the source of the information (i.e., location, status, underlying technology) semantic interoperability should be supported. Semantic interoperability can be enabled by implementing an information system that manipulates the data formats and its metadata using a formal language (i.e., JSON-LD, RDF, OWL) thus allowing dedicated components to translate-interpret the data and abstract the necessary information for its use on specific purposes.

\section{Gateway, Edge Computing, Cloud Computing}

\section{A. Gateway capabilities and edge computing}

The gateway can be running at the device layer or at the network layer having the role to connect devices using P2P or VPN protocols.

Work was carried out by our team for building a smart gateway that collects data from sensors and uses it to change the physical environment (e.g., lights, room temperature, sound) or the virtual environment (e.g., exergames) to enrich the physiotherapy session. Also, different approaches to join and synchronize the data from different wireless sensor networks (WSN) were considered. Several solutions were implemented, including real-time computation platforms of smart nodes like the ESP32 (ESP32 has Xtensa ${ }^{\circledR}$ Dual-Core 32-bit LX6 microprocessors, which runs up to 600 DMIPS). The ESP32 runs on breakout boards and modules from 160
Mhz up to $240 \mathrm{MHz}$ as WSN nodes supporting sensing and control tasks. Raspberry Pi and NI MyRIO were used as coordinators of the smart gateway system.

In our work, the WSN contains several nodes with different types of sensors and controllers that allow the acquisition and measurement of different parameters associated with user movements or physiological parameters during physical rehabilitation session (e.g., inertial measurements unit, force sensors from insole or force platforms, Leap Motion, Kinect, Shimmer ECG or PPG). The coordinator collects the data from WSN nodes and sends it to Firebase, a Google's cloud hosted no-SQL real-time database, over the internet. To avoid losing data when the internet is down, the acquired data is always saved on a local file (hosted on a SD card in the case of Raspberry Pi).

The game (based on VR -Virtual Reality) computation platform that includes a miniPC connected to the Natural User Interface (Kinect v1, Kinect v2 or Leap Motion Controller) transmits information to the smart gateway coordinator through Wi-Fi. An Application Programming Interface (API) was developed to support the dialogue between the coordinator and the VR platform. A Web application was also developed to analyze the recorded data, to control the physical environment (e.g., lighting system and the HVAC system), to tune settings of physiotherapy sessions, and implement alert mechanisms.

The applications for Smart Gateway should work on different platform, enabling its use by the users through its personal computer, laptop, tablet or smartphone.

\section{B. Server, cloud server, hybrid cloud or microservices}

The server or cloud server is responsible for storing all the information regarding data of patients, rehabilitation sessions, and exercise results. The LAMP (Linux operational system, Apache web server software; MySQL database component, PHP programming language) architecture was used. The main objective of the server or cloud server is to centralize all information in one place and can be accessed remotely by system applications. By storing this data in a server, or cloud server respectively, through a mobile application that can be used by the physiotherapists or the system manager, relevant information would be possible to be obtained after complex processing of data.

The Application Support Layer includes different components: the serious game engine based on Unity3D; the mobile applications; and the database. Unity3D is a multiplatform game engine developed by Unity Technologies. It is used for the development of video games for computers, consoles, mobile devices and websites using languages such as $\mathrm{C}++, \mathrm{C}$, Python, JavaScript and Action Script 3. It is able to support 2D and 3D graphics and can be associated with different computation devices such as mobile (smartphone and tablets), PC and PS4. Unity3D performs well when connecting to a cloud server to store and retrieve game-related data. The Unity3D platform is increasingly being used by developers due to its powerful game engine, but other game makers platforms could be used as well (e.g., Unreal engine or the Game Maker studio).

The implemented database allows to register a new user and introduce demographic and biometric data (e.g., name, gender, age, height, weight). The data regarding the games and exercises done by the patient (i.e., number of movements 
repetition, number of times played, scores) are also stored. Different metrics were calculated that can be used by the physiotherapists for objective evaluation of the rehabilitation process (e.g., metrics for gait characterization, angle of upper limbs movements, energy expenditure, heart rate variability). The game customization parameters can be accessed from the game interface or from the mobile application, either by the patient or by the physiotherapist. The Web site, localized in a server or a cloud server, has different levels of access depending on the type of client: administrator, physiotherapist or patient. The administrator has full access to the information system (including account management) in order to carry out maintenance or upgrade procedures. The physiotherapist is able to access all the data stored in the database related with their patients, add complementary information (such as results from questionnaires, medical records, opinions from other colleagues and free notes), and to choose the rehabilitation program for a given patient. The physiotherapist is also able to tune the rehabilitation program by making adjustments on key parameters, and to act directly on the virtual-reality generator bypassing the decisions taken by the smart gateway. The patient has access to its own data (scored from game, the realized physiotherapy session, outcomes).

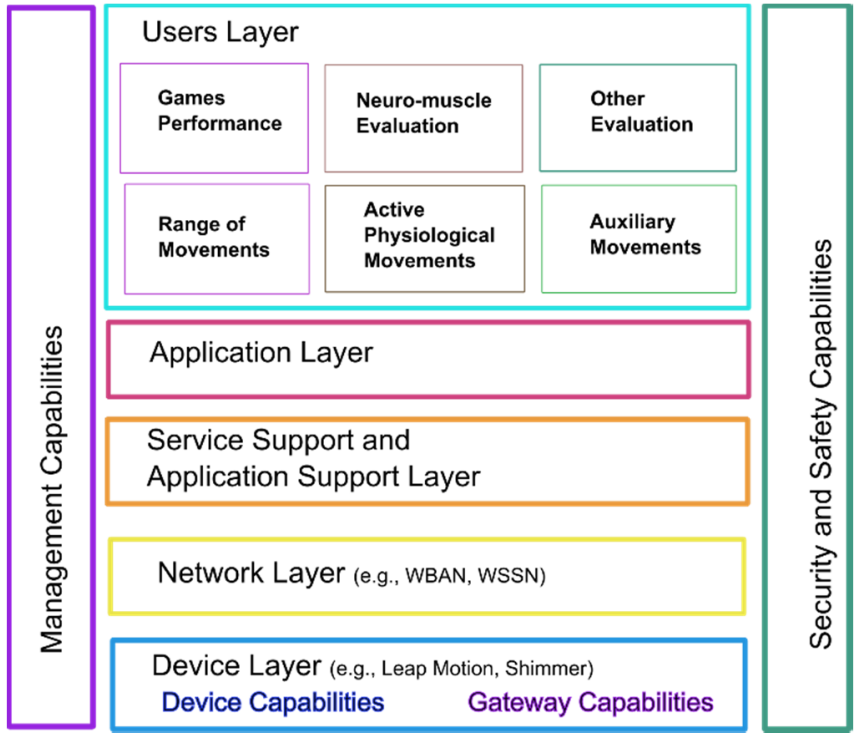

Fig. 1. IoT based architecture of information system for physiotherapy

In the system developed by our team the Web interface includes REST web services to interact with third-party applications, including the smart gateway. Any client application (desktop or mobile, Windows or Linux), after successful authentication, can connect to these services and consume the methods they expose.

Different mobile applications using Android or iOS may be integrated in the IoT system for data visualization and for serious game setting for different physiotherapy plan. Fig. 1 shows a model of the designed architecture following a function architecture that refers to ITU-T Y.2060 [17].

A server, cloud server, hybrid cloud or microservices might be adopted for architecture of IoT based information system, for computing, storage, analytics and data visualization. In our work, prototypes were realized using a server and a cloud server, respectively. Cloud computing may provide functional and non-functional support (i.e., Service Level Agreements, low latency fault-tolerance, costoptimization, security). These capabilities are provided as services (platform-as-a-service, infrastructure-as-a-service, software-as-a-service, etc.). Hybrid cloud blends internal information system with cloud powered services. It might integrate private, public and managed cloud with the existing on-premises information system (e.g., Fujitsu MetaArc and Cloud Service K5). This may improve the agility, accessibility and security of the information system for physiotherapy, by providing higher level of sensible data security.

Both the ETSI team [20] and the Microsoft Azure team [21] recommend the use of microservices in IoT architecture. Microservices are an architectural approach for developing applications as a set of small services, where each service is running as a separate process, communicating through simple mechanisms [20]. The main advantage of microservice-based architecture is that one can develop, deploy, upgrade and scale every microservice independently of the others. Moreover, developing microservices separately enable the use of different technologies for each microservice and to have distinctive microservices for devices that communicate using different protocols. Also, this may support the safety and security of the system. One drawback of this approach is that microservices use quite recent communication mechanisms that might be not supported by the legacy systems.

Reference architectures for IoT have been proposed by several working groups like the IoT-A European project (Internet of Things Architecture), ETSI, Microsoft Azure team, or the CREATE-IoT European project [17]. The ETSI team identified several functional and non-functional requirements for IoT (i.e., multi-tenancy, massive data processing, high-throughput, high-availability, low latency, security). A single instance of a software application should serve multiple customers (multi-tenancy). When all of customer data is handled using the same software resources, the architecture design should be extremely rigorous and prevent data leakage [19]. High volume and high velocity data are produced. These data should be analyzed and used to trigger action in IoT systems (massive data processing). The processing of data can be done in real time (streaming) or is collected over time and then fed into an analytics system (batch processing model). By building data streams, one can feed data into analytics tools as soon as it is generated, and get near-instant analytics results. For streaming data processing, complex event processing (CEP) might be used with the goal to identify significant events and respond fast. Batch processing is adequate in situations where non-realtime analytics results is needed, and when it is more important to process large volume of information than to get fast analytics results.

Services provided by IoT should be resilient to failures (i.e., restore functionalities easily when the service host crashes) and be able to restart on another machine (highavailable applications). Such failures can be produced by a service-internal problem or during an application upgrade. The running service should determine whether it can continue to move forward to the newer version or to roll back to a previous stable version to maintain a consistent state. The service should be designed so that the process can be restarted at any time with no data loss. Low latency is also important 
requirement for IoT. The ETSI team suggested that this can be achieved by: brokers capable of routing IoT application messages in (near) real time; MapReduce, which can make use of massive in-memory databases capable of meeting lowlatency requirements; and edge computing that allows to reduce network latency by moving computing resources closer to the field domain where an action takes place [20].

Advanced technologies facilitate moving computation closer to the source by embedding computation near or within the sensor and the memories. The use of memristors for analog computation and 3-D stacked memory might also help on complex signal processing in edge computing.

An important challenge for an IoT-based information system for healthcare is the security of the system. The security and privacy of data are important issue in developing IoT based information system for physiotherapy (i.e.., taking into account the amount of personal data that can be acquired by information systems, as well as the capacity of the analytics tools to obtain more personal data by data triangulation and the use of artificial intelligence). Nowadays, information systems are susceptible to malicious usage by hackers but also by unauthorized competitors. Therefore, security of information system (i.e., protection of the confidentiality, integrity and availability of information asset) has a great importance. Different strategies should be investigated to implement security at every layer of the architecture of information system for healthcare based on IoT.

\section{CONCLUSION}

The amount of data that can be acquired using advances in sensor and IoT technologies, and the information provided by analytics tool in IoT frameworks are enormous. There are few studies about information systems for physiotherapy. Moreover, scarce data exists related to integration of IoT technology into information systems for physiotherapy or motor rehabilitation. The data acquired by small devices at home, clinics or hospitals, which gives information about movements, activities and the general physical condition of the patient, may increase the accessibility to healthcare services and the quality of care in a safe environment. More research is needed for better understanding what are the optimal model and architecture for IoT platforms for people having different type of disabilities, as well as an optimal universal design that may increase the quality of care for disabled people. Taking into account the diversities of functional impairments, the great challenge of IoT for physiotherapy or motor rehabilitation is to create and implement an adaptive framework, having capacity for multimodalities sensing, modularity, adequate and safe connectivity to the existing information systems, and which processes enormous quantity of data. As IoT data and information are nowadays susceptible to malicious usage by hackers but also by unauthorized competitors, design of information systems that support security of healthcare information at every layer of the information system architecture also is a challenge for developers, leading to rethink many of the previous developed healthcare models.

\section{ACKNOWLEDGMENT}

The work was supported by Fundação para a Ciência e a Tecnologia and Instituto de Telecomunicações, in the framework of the project TailorPhy-Intelligent Sensors and Tailored Environments for Physiotherapy, PTDC/DTPDES/6776/2014.

\section{REFERENCES}

[1] T. Szentes, I. Vályi-Nagy. "Restructuring the healthcare system: redesigning based on needs," Orv. Hetil., vol. 156(3), pp. 83-91, 2015.

[2] S.M. Hosseini Shokouh, M. Arab, S. Emamgholipour, A. Rashidian, A. Montazeri, R. Zaboli. "Conceptual models of social determinants of health: a narrative review," Iran J. Public Health, vol. 46(4), pp. 435-446, 2017.

[3] G.L. Engel. "The need for a new medical model: a challenge for biomedicine," Science, vol. 196(4286), pp. 129-136, 1977. https://www.physio-pedia.com/Biopsychosocial_Model

[4] A. Roman. "The holistic model of health and methods of its evaluation," J. Prev. Medicine $-9^{\text {th }}$ Edition of International Conference on Preventive Medicine and Public Health, 2018 http://www.imedpub.com/proceedings/the-holistic-model-ofhealth-and-methods-of-its-evaluation-3892.html

[5] European Physical and Rehabilitation Medicine Bodies Alliance. White book on physical and rehabilitation medicine in Europe. Chapter 2. "Why rehabilitation is needed by individual and society". European J. Phys. Rehab. Medicine, vol. 54(2), pp. 166-176, 2018.

[6] World Health Organization (WHO). Integrated care models: an overview, pp. 1-42, October 2016.

[7] R. Hébert, P.J. Durand, N. Dubuc, A. Tourigny, The PRSIMA Group. "PRISMA: a new model of integrated service delivery for the frail older people in Canada". International Journal of Integrated Care, vol. 3, pp. 1-8, 2003.

[8] Veterans Affairs: https://www.ehrm.va.gov/

[9] S.L. DuVall, M.E. Matheny, I.R. Ibragimov, T.D. Oats, J.N. Tucker, B.R. South, A. Turano, H. Saoudian, C. Kangas, K. Hofmann, W. Funk, C. Nichols, A. Bonnema, L. Ferrucci, J.R. Nebeker. "A Tale of Two Databases: The DoD and VA Infrastructure for Clinical Intelligence (DaVINCI)". Studies in Health Technology and Informatics, vol. 264, MEDINFO 2019: Health and Wellbeing e.Networks for All, pp. 16601661, 2019.

[10] Sustainable Development Unit: https://www.sduhealth.org.uk/

[11] Australian Medical Association: https://ama.com.au/positionstatement/environmental-sustainability-health-care-2019

[12] C. Gutenbrunner, A.B. Ward, M.A. Chamberlain. "The white book on physical and rehabilitation medicine in Europe," J. Rehabil. Med., pp. 287-333, 2006.

[13] C. Gutenbrunner, A. Ward, M. Chamberlain. "The white book on physical and rehabilitation medicine in Europe," J. Rehabil. Med. (45 Suppl), 2007.

[14] L. Turner-Stokes, H. Williams, A. Bill, P. Bassett, K. Sephton. "Cost-efficiency of specialist inpatient rehabilitation for working-aged adults with complex neurological disabilities: a multicentre cohort analysis of a national clinical data set," BMJ Open, vol.6(2):e010238, 2016.

[15] D.J. Reinkensmeyer, S. Blackstone, C. Bodine, J. Brabyn, D. Brienza, K. Kevin Caves, F. DeRuyter, E. Durfee, S. Fatone, G. Fernie, S. Gard, P. Karg, T.A. Kuiken, G.F. Harris, M. Jones, Yue Li, J. Maisel, M. McCue, A. Michelle, M.A. Meade, H. Mitchell, T.L. Mitzner, J.L. Patton, P.S. Requejo, J.H. Rimmer, W.A. Rogers, W. Zev Rymer, J.A. Stanford, J.A. Schneider, L. Sliker, S. Sprigle, A. Steinfeld, E. Steifeld, G. Vanderheiden, C. Winstein, L.Q. Zhang, T. Corfman. How a diverse research ecosystem has generated new rehabilitation technologies: Review of NIDILRR's Rehabilitation 
Engineering Research Centers. J. NeuroEngineering and Rehabil, vol. 14(109), pp. 1-53, 2017.

[16] Interiot. Interoperability of Heterogeneous IoT Platforms, 2016.

[17] CREATE-IoT. Cross Fertilization through Alignment, Synchronization and Exchanges for IoT, 2018.

[18] WHO. Disabilities. https://www.who.int/topics/disabilities/en/
[19] WHO. Disability. https://www.who.int/disabilities/en/

[20] ETSI Technical Report TR103527. SmartM2M; Virtualized IoT Architectures with Cloud Back-ends. https://etsi.org/newsevents

[21] Microsoft Azure IoT Reference Architecture. https://azure.microsoft.com/en-us/blog/azure-iot-referencearchitecture-update 